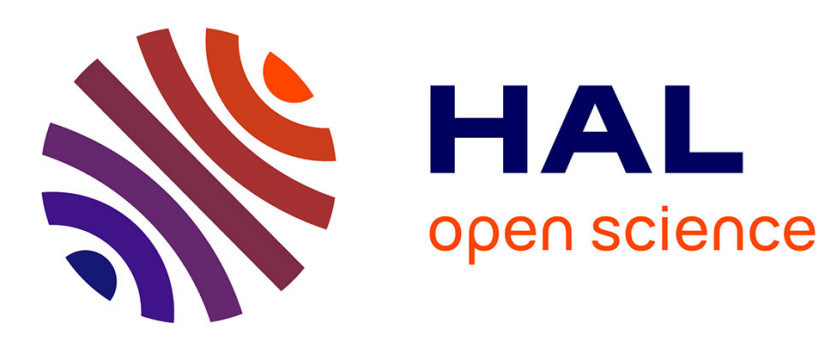

\title{
Conformal invariant saturation
}

H. Navelet, R. Peschanski

\section{- To cite this version:}

H. Navelet, R. Peschanski. Conformal invariant saturation. Nuclear Physics B, 2002, 634 (1-2), pp.291-308. 10.1016/S0550-3213(02)00314-0 . cea-02892007

\section{HAL Id: cea-02892007 https://hal-cea.archives-ouvertes.fr/cea-02892007}

Submitted on 7 Jul 2020

HAL is a multi-disciplinary open access archive for the deposit and dissemination of scientific research documents, whether they are published or not. The documents may come from teaching and research institutions in France or abroad, or from public or private research centers.
L'archive ouverte pluridisciplinaire HAL, est destinée au dépôt et à la diffusion de documents scientifiques de niveau recherche, publiés ou non, émanant des établissements d'enseignement et de recherche français ou étrangers, des laboratoires publics ou privés. 


\title{
Conformal invariant saturation
}

\author{
H. Navelet and R. Peschanski*
}

\begin{abstract}
We show that, in onium-onium scattering at (very) high energy, a transition to saturation happens due to quantum fluctuations of QCD dipoles. This transition starts when the order $\alpha^{2}$ correction of the dipole loop is compensated by its faster energy evolution, leading to a negative interference with the tree level amplitude. After a derivation of the the one-loop dipole contribution using conformal invariance of the elastic 4-gluon amplitude in high energy QCD, we obtain an exact expression of the saturation line in the plane $(\mathrm{Y}, \mathrm{L})$ where $\mathrm{Y}$ is the total rapidity and $\mathrm{L}$, the logarithm of the onium scale ratio. It shows universal features implying the Balitskyi - Fadin - Kuraev - Lipatov (BFKL) evolution kernel and the square of the QCD triple Pomeron vertex. For large L, only the higher BFKL Eigenvalue contributes, leading to a saturation depending on leading log perturbative QCD characteristics. For initial onium scales of same order, however, it involves an unlimited summation over all conformal BFKL Eigenstates. In all cases, conformal invariance is preserved for the saturation mechanism based on dipole loops.
\end{abstract}

\section{SATURATION FROM QCD DIPOLES}

A regime of QCD at small coupling constant where the density of partons begins to saturate has since long been the subject of many studies [1 18]. In one class of models, one expects the effect due to the high energy multiplication of partons due to the QCD dynamics. Another way which was proposed to investigate high density partonic effects is the consideration of collisions at high energy on heavy nuclei. Indeed, the number of partons is supposed to be large in the background of the collision providing boundary conditions favorable for the saturation mechanism to happen. In the present paper, we will focus on the first scheme, i.e. the saturation mechanism due to the energy evolution of the parton density in a purely perturbative QCD framework. More precisely, we will consider even "perturbative QCD" boundary conditions, in order to select the features of saturation which could be fully calculated from the QCD lagrangian.

On the experimental ground, saturation has not been clearly identified in the evolution of structure functions in the HERA range, even if some evidence for partial saturation has been found by analyzing vector meson diffractive production in impact parameter space [19]. The problem of identifying saturation effects in actual or future experiments is very stimulating, since the increase of the energy range is at reach at Tevatron and later at LHC. Even, some lessons will be available from experiments with heavy nuclei at RICH. The goal of our purely perturbative calculations, even if they appear to be far from the experimental realizations, is to give an example of saturation which could be fully computed, without referring to a non-perturbative ansatz. In practice, later on, it will be possible, using factorization properties, to combine our calculations with some reasonable non-perturbative wave functions to get predictions for realistic processes, like hard hadron-hadron interations at Tevatron and LHC.

Our idea is to start from the most elementary hard processes, namely onium-onium scattering at (very) high energies. The reaction can be described [4,5] by the wave-functions of the onia in terms of $q \bar{q}$ colour singlet dipoles at the time of the (hard) interaction, which then interact through double gluon exchanges. This description, which corresponds to the tree level dipole interactions, is equivalent to the usual Balitsky, Fadin, Kuraev, Lipatov (BFKL) formalism [20] for the same process, as can be proven [21] using the conformal invariant symmetry of the BFKL evolution equation. When energy increases, the wave functions has an ever increasing density of colour dipoles and the tree level dipole interaction is expected to be modified by unitarity corrections which are already present in the perturbative regime. The first order correction is the one-loop dipole contribution which was first considered in Ref. 22] as a formal calculation of next-to-next order contributions in the coupling constant. In the present paper we show that, despite its higher order, this one-loop unitarity correction gives rise to the onset of saturation in oniumonium scattering and thus allows one to give a complete perturbative QCD description of the saturation critical line. This saturation mechanism is to be distinguished from the one which would come from the interaction of a onium (e.g. a collection of independent dipoles) with a large nucleus (see, e.g. 8, 11 13, 15, 17, 18]), which, in some cases, can be associated with a non-linear branching process without dipole recombination. In the case we consider, the non-linearities due to the high density of partons correspond to the recombination of QCD dipoles.

${ }^{*}$ CEA, Service de Physique Theorique, CE-Saclay, F-91191 Gif-sur-Yvette Cedex, France 
We write the onium-onium elastic cross-section

$$
\frac{d \sigma}{d Q^{2}}=\frac{1}{4 \pi}\left|F_{Q}\right|^{2}
$$

where $Q$ is the transfer momentum. The amplitude reads

$$
F_{Q}=-i \int d^{2} \rho d^{2} \rho^{\prime} \int_{0}^{1} d z d z^{\prime} \Phi(\rho, z) \Phi\left(\rho^{\prime}, z^{\prime}\right) f_{Q}\left(\rho, \rho^{\prime} \mid Y\right)
$$

where the onium is defined as a heavy $\bar{q} q$ state with $q$ (resp. $\bar{q}$ ) having the fraction $z$ (resp. $1-z$ ) of the longitudinal momentum in an infinite momentum frame. It is defined by its wave-function $\Phi(\rho, z)$ over a basis of dipole states of transverse size $\rho \equiv \rho_{0}-\rho_{1}$, where $\rho_{0}, \rho_{1}$ are the positions of the dipole's constituants in transverse coordinate space. $f_{Q}\left(\rho, \rho^{\prime} \mid Y\right)$ is the dipole-dipole elastic amplitude for a full rapidity interval $Y$ and a momentum transfer $Q$, the calculation of which at one dipole-loop order and saturation properties are the subject of the present paper.

In fact the physical origin of the saturation mechanism that we want to investigate is the following: it is known [4 that the wave function of an Onium in the infinite momentum frame develop a large number of dipole states as a function of the rapidity (which plays the rôle of time in a cascading mechanism). However, the larger is the dipole number, the smaller is their size and thus they do not contribute to saturation. This can be proven rigorously by the fact that the dipole-dipole cross-section add together to give back the BFKL cross-section 21]. It is interesting to note the use of conformal invariance to get this exact bootstrap property between an "s-channel" and "t-channel" description of the same amplitude.

However, now if one computes the dipole-dipole interaction at one-loop level, the system is allowed to take into account quantum fluctuations where virtual dipole pairs may be created during the time of interaction. Moreover, all sizes of dipoles can contribute in the loop, which goes beyond the limitations of the tree-level mechanism. This is expected from perturbative unitarity arguments 湫 which expect the damping of the energy dependence of the amplitude by saturation effects due to multi-loop dipole contributions. This is precisely the goal of our work to put this expectation on a quantitative level.

Through unitarity arguments, the QCD saturation phase corresponding to the mechanism we consider requires an estimate of all dipole loop contributions, at least in the high energy approximation. In the present paper we will concentrate on the first step, i.e. the transition to saturation which is expected to be due to the interfernece between the tree and 1-loop level. We expect that the techniques which we were led to consider in this work, relying notably on the conformal invariance properties, will be useful to investigate this intringing phase of matter.

Our main results are the following:

i) Up to one-loop QCD dipole level, the dipole-dipole amplitude reads

$$
\begin{aligned}
f_{Q}\left(\rho, \rho^{\prime} \mid Y\right) & \equiv f_{Q}^{(0)}+f_{Q}^{(1)}=\frac{\alpha^{2}}{4}\left|\rho \rho^{\prime}\right| \int d h \bar{E}_{Q}^{h}\left(\rho^{\prime}\right) E_{Q}^{h}(\rho) d(h) e^{\omega(h) Y} \\
& -\left(\frac{2 \alpha}{\pi^{4}}\right)^{4} \frac{\pi e^{2 \omega\left(\frac{1}{2}\right) Y}}{\left[\omega^{\prime \prime}\left(\frac{1}{2}\right) Y\right]^{3}}\left|\rho \rho^{\prime}\right| \int d h(-)^{n} \bar{E}_{Q}^{h}\left(\rho^{\prime}\right) E_{Q}^{h}(\rho)\left|\frac{g_{3 \mathcal{P}}\left(h, \frac{1}{2}, \frac{1}{2}\right)}{\chi(h)-2 \chi\left(\frac{1}{2}\right)}\right|^{2}
\end{aligned}
$$

In equation (3), the symbolic notation $\int d h \equiv \sum_{n=-\infty}^{\infty} \int d \nu$ corresponds to the integration over the quantum numbers associated to the continuous unitary irreducible representations of $S L(2, \mathcal{C})$, namely

$$
h=i \nu+\frac{1-n}{2} ; \tilde{h}=i \nu+\frac{1+n}{2} ; n \in \mathcal{N}, \nu \in \mathcal{R} .
$$

The $S L(2, \mathcal{C})$ Eigenvalue of the BFKL kernel is

$$
\omega(h)=\frac{\alpha N_{c}}{\pi} \chi(h) \equiv \frac{\alpha N_{c}}{\pi}\{2 \Psi(1)-\Psi(h)-\Psi(1-\tilde{h})\},
$$

where $\Psi \equiv(\log \Gamma)^{\prime}$, and $E_{Q}^{h}(\rho)$ are the $S L(2, \mathcal{C})$ Eigenfunctions [23]. $g_{3 \mathcal{P}}\left(h, h_{a}, h_{b}\right)$ is the known QCD triple Pomeron coupling function [24.25], whose definition is given in Appendix 1, together with $E_{Q}^{h}(\rho)$ and the other ingredients (such as the functions $a(h), d(h)$ ) of the $S L(2, \mathcal{C})$-invariant formalism.

The symmetry of the tree level amplitude remains valid at one-loop level, leading to a conformal invariant saturation scheme. 
ii) The evaluation of formula (3) when the condition $L \equiv \log \left|\frac{\rho}{\rho^{\prime}}\right| \gg 1$ is fulfilled can be done in the forward direction $(Q=0)$ in terms of the leading Eigenfunction and Eigenvalue of the BFKL kernel, namely with $n=0$. By contrast, for similar dipole sizes $(L=\mathcal{O}(1))$ infinitely many Eigenfunctions and Eigenvalues contribute to the loop expression, leading to a quite different structure.

The QCD critical saturation line is derived in the $L \gg 1$ regime and is given by:

$$
\tilde{Y}\left(\frac{\chi\left(h^{*}\right)-\chi\left(h_{c}\right)}{\chi\left(\frac{1}{2}\right)}\right)-2 L\left(h_{c}-h^{*}\right)=\tilde{Y}_{c}+\frac{5}{2} \log \tilde{Y}-\log L,
$$

where $\tilde{Y} \equiv \omega(1 / 2) Y$ and $\tilde{Y}_{c} \sim \mathcal{O}\left\{\log \left(1 / \alpha^{2}\right)\right\}$ is a large constant (formula (16) in the text). The transition to saturation occurs when the first member of (6) exceeds the second one. Note that using the variable $\tilde{Y}$ means that the rapidity (i.e. energy) dependence of the amplitude is quantified by reference to the BFKL intercept $1+\omega(1 / 2)$. Hence the overall $\tilde{Y}, L$ dependence is determined by "universal" numbers, independent of the coupling constant.

In formula (6), there appears two critical values of the effective anomalous dimensions $h_{c}$ for the tree level and $h^{*}$ for the one-loop contribution. They are both defined as solution of implicit equations

$$
\frac{\chi^{\prime}\left(h_{c}\right)}{\chi\left(\frac{1}{2}\right)}=\frac{2 L}{\tilde{Y}} ; \quad \chi\left(h^{*}\right)=2 \chi(1 / 2) .
$$

$h_{c}$ is a moving saddle point in the tree-level integrand of (3) while $h^{*} \sim .183$ is the fixed location of a double pole singularity in the one-loop integrand of (3).

The plan of the paper is the following. The next section II is devoted to the derivation of formula (3) and Section III to that of formula (6). A disussion of the resulting saturation line and an outlook are proposed in section IV. The $S L(2, \mathcal{C})$-invariant formalism which we use is presented in Appendix 1 and some technical parts of the derivation of the one-loop amplitude are present in Appendix 2 and 3.

\section{THE ONE-LOOP DIPOLE AMPLITUDE}

At one-loop level, the amplitude $f_{Q}^{(1)}$ has a simple and physically appealing formulation [⿴囗十 in the transverse coordinate space, see Fig.1. We write

$$
f_{Q}^{(1)}\left(\rho, \rho^{\prime} \mid Y\right) \equiv \frac{1}{2 \pi} \int d^{2} r e^{i Q} T^{(1)}\left(\rho_{0} \rho_{1} ; \rho_{0}^{\prime} \rho_{1}^{\prime} \mid Y\right)
$$

with impact parameter $r$. The dipole-dipole amplitude in coordinate space reads

$$
\begin{gathered}
T^{(1)}\left(\rho_{0} \rho_{1} ; \rho_{0}^{\prime} \rho_{1}^{\prime} \mid Y=y+y^{\prime}\right) \equiv-\int_{0}^{y} d \bar{y} \int_{0}^{y^{\prime}} d \bar{y}^{\prime} \int \frac{d^{2} \rho_{a_{0}} d^{2} \rho_{a_{1}} d^{2} \rho_{b_{0}} d^{2} \rho_{b_{1}}}{\left|\rho_{a} \rho_{b}\right|^{2}} \frac{d^{2} \rho_{a_{0}^{\prime}} d^{2} \rho_{a_{1}^{\prime}} d^{2} \rho_{b_{0}^{\prime}} d^{2} \rho_{b_{1}^{\prime}}}{\left|\rho_{a^{\prime}} \rho_{b^{\prime}}\right|^{2}} \\
\times n_{2}\left(\rho_{0} \rho_{1} ; \rho_{a_{0}} \rho_{a_{1}}, \rho_{b_{0}} \rho_{b_{1}} \mid y-\bar{y}, \bar{y}\right) \bar{n}_{2}\left(\rho_{0}^{\prime} \rho_{1}^{\prime} ; \rho_{a_{0}}^{\prime} \rho_{a_{1}}^{\prime}, \rho_{b_{0}}^{\prime} \rho_{b_{1}}^{\prime} \mid y^{\prime}-\bar{y}^{\prime}, \bar{y}^{\prime}\right) T\left(\rho_{a_{0}} \rho_{a_{1}}, \rho_{a_{0}^{\prime}} \rho_{a_{1}^{\prime}}\right) \bar{T}\left(\rho_{b_{0}} \rho_{b_{1}}, \rho_{b_{0}^{\prime}} \rho_{b_{1}^{\prime}}\right)
\end{gathered}
$$

where $\rho_{0}, \rho_{1}$ are the transverse coordinates of one of the initially colliding dipoles (resp. $\rho_{0}^{\prime}, \rho_{1}^{\prime}$ for the second one), while $\rho_{a_{0}} \rho_{a_{1}}$ and $\rho_{b_{0}} \rho_{b_{1}}$, are the two interacting dipoles emerging from the dipole $\rho_{0} \rho_{1}$ after evolution in rapidity (resp. $\rho_{\{i\}} \rightarrow \rho_{\left\{i^{\prime}\right\}}$, for the second one).

The overall minus sign comes from the well-known AGK rules [26] relating the amplitudes to the discontinuity here calculated in the c.o.m. frame of the dipole-dipole reaction. The tree level interaction amplitudes $T$ are given in Appendix 1. In formula (9), $n_{2}\left(\rho_{0} \rho_{1} ; \rho_{a_{0}} \rho_{a_{1}}, \rho_{b_{0}} \rho_{b_{1}} \mid y-\bar{y}, \bar{y}\right)$ stands for the probability of producing two dipoles after a mixed rapidity evolution [4] 22], namely with a rapidity $y-\bar{y}$ with one-Pomeron type of evolution and a rapidity $\bar{y}$ with two-Pomeron type of evolution, see Fig.2. Further on, one has to integrate over $\bar{y}, \bar{y}^{\prime}$. The solution for $n_{2}$ is given in Appendix 2. The integration over intermediate variables yields a drastic simplification due to the appearance of quite a few $\delta$-functions. This tedious but straightforward derivation is given in detail in Appendix $\mathbf{3}$.

Starting with the general formula (A.25) obtained in Appendix 3, we first evaluate the high energy behaviour of the integral over $h_{a, b}$ the dominant contribution of which, as well-known from the ordinary BFKL analysis, is obtained for $n_{a, b}=0$. The integrals are dominated by the usual BFKL saddle-points at $h_{a, b}=\frac{1}{2} ; \omega\left(\frac{1}{2}\right)=4 \log 2$. Taking into 
account in the saddle-point derivation the logarithmic corrections due to the zeroes in the prefactors at $h_{a, b}=\frac{1}{2}$, one gets:

$$
f_{Q}^{(1)}\left(\rho, \rho^{\prime} \mid Y\right) \sim-\left(\frac{2}{\pi}\right)^{8}\left(\frac{\alpha}{2 \pi^{2}}\right)^{4} \frac{\pi e^{2 \omega\left(\frac{1}{2}\right) Y}}{\left[\omega^{\prime \prime}\left(\frac{1}{2}\right) Y\right]^{3}}\left|\rho \rho^{\prime}\right| \int d h(-)^{n} \bar{E}_{Q}^{h}\left(\rho^{\prime}\right) E_{Q}^{h}(\rho)\left|\frac{g_{3 \mathcal{P}}\left(h, \frac{1}{2}, \frac{1}{2}\right)}{\chi(h)-2 \chi\left(\frac{1}{2}\right)}\right|^{2} .
$$

Formula (10) is then reported in the full amplitude (3).

A few comments on our result (10) are in order:

i) Conformal invariance is explicitly realized, due to the decomposition on the combination of $S L(2, \mathcal{C})$ Eigenvectors $\bar{E}_{Q}^{h}\left(\rho^{\prime}\right) E_{Q}^{h}(\rho)$. This property being shared by the tree-level amplitude, see (A.1), is thus valid for the whole amplitude (3) including one-loop level. Hence, saturation which is expected from the compensation between tree level and one-loop due to the different energy dependence is genuinely a conformal invariant phenomenon. We conjecture that this will be true to all orders, since only tensorial $S L(2, \mathcal{C})$ properties play a rôle in the symmetry properties of the amplitude. Thus the saturation regime based on dipole loop contribution, if mathematically convergent, is expected to respect the conformal symmetry.

ii) The key difference between the tree-level and one-loop amplitude properties lies in the difference in the analytic singularities of the integrands. While the tree level term in (3) is dominated by a moving saddle-point at high energy, the one loop amplitude (10) has double poles at $\chi(h)=2 \chi(1 / 2)=8 \log 2$ which depend only on the anomalous dimensions $h$ and thus are independent of the energy. This very peculiar property means that the one-loop contribution may depend (and thus can give information) on BFKL properties which are not reached in the conventional analysis. In particular, all higher conformal spins may contribute, contrary to the tree level contribution. Also, contributions for values of $h$ not limited to the interval $\left[0^{+}, \frac{1}{2}\right]$ can give rise to poles. This may be interpreted as unlimited contributions of higher twist terms of the BFKL solution.

iii) Apart the factor $\alpha^{4}$, which is the natural strength scale of the one-loop amplitude, there is a dynamical factor connected to the square of the function $g_{3 \mathcal{P}}\left(h, \frac{1}{2}, \frac{1}{2}\right)$. This function is the QCD analogue of the triple Pomeron coupling. However, it is not the value $g_{3 \mathcal{P}}\left(\frac{1}{2}, \frac{1}{2}, \frac{1}{2}\right)$ which is here relevant, as was the case in the QCD dipole analysis of the triple pomeron coupling in 25, since $h=\frac{1}{2}$ is not solution of the double pole equation. One could also note that the square of $g_{3 \mathcal{P}}$ appears, contrary to saturation equations on an extended target, where the dependence is linear [11].

\section{SATURATION CRITICAL LINE}

As discussed in section I, saturation is expected to take place when the one-loop amplitude, with coupling of order $\alpha^{4}$, becomes comparable in strength with the tree level amplitude of order $\alpha^{2}$. Formula (3) is completely explicit and thus can serve for analyzing saturation depending on transverse momentum or, equivalently using the Fourier transforms (A.4), on impact parameter. For sake of simplicity we will concentrate on the forward contribution at $Q=0$, that is (through unitarity) for the total cross-section integrated over impact parameter. In this limit, the $S L(2, \mathcal{C})$ Eigenvectors simplify [23] to give

$$
\bar{E}_{Q}^{h}\left(\rho^{\prime}\right) E_{Q}^{h}(\rho) \rightarrow\left(\frac{\rho}{\rho^{\prime}}\right)^{h-\frac{1}{2}}\left(\frac{\bar{\rho}}{\bar{\rho}^{\prime}}\right)^{\tilde{h}-\frac{1}{2}} .
$$

Consequently, we may infer that the key "order parameter" for the discussion of saturation will be the scale invariant ratio of incident dipole sizes $\rho / \rho^{\prime}$, in conformity with the conformal invariance of the amplitude. This will in turn be determined by the ratio of the characteristic scales of the incoming systems which are encoded in their corresponding QCD dipole wave functions, see (2).

The general solution of (3) is easily obtained by a summation over the double pole contributions verifying $\chi\left(h_{i}\right)=$ $2 \chi\left(\frac{1}{2}\right)$, whose location are caracterized by an infinite number of values $\left.h_{i}\right)=\left(n_{i}, \gamma_{i}\right)$. One finds a formal double series

$$
\begin{aligned}
f_{Q=0}^{(1)}\left(\rho, \rho^{\prime} \mid Y\right) & \sim-\left(\frac{2}{\pi}\right)^{6}\left(\frac{\alpha}{\pi^{2}}\right)^{4} \frac{e^{2 \omega\left(\frac{1}{2}\right) Y}}{\left[\omega^{\prime \prime}\left(\frac{1}{2}\right) Y\right]^{3}} \\
& \times \sum_{n_{i}, \gamma_{i}}(-1)^{n}\left|\frac{g_{3 \mathcal{P}}\left(h, \frac{1}{2}, \frac{1}{2}\right)}{\chi^{\prime}\left(h_{i}\right)}\right|^{2}\left(\frac{\rho}{\rho^{\prime}}\right)^{h_{i}}\left(\frac{\bar{\rho}}{\bar{\rho}^{\prime}}\right)^{\tilde{h}_{i}}\left\{L+\frac{\partial \log \left|g_{3 \mathcal{P}}\left(h, \frac{1}{2}, \frac{1}{2}\right)\right|}{\partial h}\right\}_{h=h_{i}},
\end{aligned}
$$


where, for sake of simplicity, we used the notation $L \equiv \log \left|\rho / \rho^{\prime}\right|$.

For incident dipoles of comparable size, $L \sim \mathcal{O}(1)$, we may note that the one loop result then depends on an infinite sum over $\left(n_{i}, \gamma_{i}\right)$ which is due to the analytic properties of the function $\chi(h)$. It even raises the question of the convergence of the double series (12). On a physical ground, since the anomalous dimensions $h_{i}$ have no obvious limitation, this situation can be interpreted as due to a dependence of the one loop amplitude on all higher twist contributions, including those with non zero conformal spins $n_{i}$. This is a quite strange example where higher twist calculations are required to all orders in a purely perturbative framework, in striking analogy with actual non perturbative situations.

Leaving this regime for further work, we will here focus on the case where only the first pole with $h^{*}=\left(\gamma_{i}^{*}, n_{i}^{*}=0\right)$ contributes, i.e. where the equivalent of a leading twist contribution is sufficient. This approximation is realized in a region where $L \gg 1$ (or $L \ll 1$ by symmetry).

The dominant double pole in (12) such that $\chi\left(h^{*}\right)=2 \chi\left(\frac{1}{2}\right)=8 \log 2$ is $h^{*} \equiv\left(n^{*}=0, \gamma^{*} \sim .183\right)$. Consequently, factorizing the tree level coupling, the resulting one loop amplitude can be written

$$
f_{Q=0}^{(1)}(L, \tilde{Y}) \sim \frac{\alpha^{2}}{4}\left|\rho \rho^{\prime}\right| \exp \left\{2 \tilde{Y}+2\left(h^{*}-\frac{1}{2}\right) L-\tilde{Y}_{1}-3 \log \tilde{Y}+\log L\right\}
$$

where we define $\tilde{Y} \equiv \omega\left(\frac{1}{2}\right) Y$, and

$$
\tilde{Y}_{1}=\log \left\{\frac{\pi^{12}}{16 \alpha^{2}}\left|\frac{\chi^{\prime}\left(h^{*}\right)}{g_{3 \mathcal{P}}\left(h^{*}, \frac{1}{2}, \frac{1}{2}\right)}\right|^{2}\left(\frac{\chi\left(\frac{1}{2}\right)}{\chi^{\prime \prime}\left(\frac{1}{2}\right)}\right)^{3}\right\}
$$

which is a priori large, being in the perturbative domain $\alpha \ll 1$ and constant up to $1 / L$ correction terms.

We define the saturation critical line as the line in the $(\tilde{Y}, L)$ where the tree-level contribution equals the one loop one. It is a simple extension to the conformal invariant configuration of the usual one [1]. Beyond this line, saturation is expected to take place.

We now make use of the well-known saddle-point approximation of the tree level amplitude

$$
f_{Q=0}^{(0)}(L, \tilde{Y}) \sim \frac{\alpha^{2}}{4}\left|\rho \rho^{\prime}\right| \exp \left\{\frac{\chi\left(h_{c}\right)}{\chi(1 / 2)} \tilde{Y}+2\left(h_{c}-1 / 2\right) L-\log \left(\frac{1}{h_{c}^{2}\left(1-h_{c}\right)^{2}} \sqrt{\frac{2 \pi \chi\left(\frac{1}{2}\right)}{\chi^{\prime \prime}\left(\frac{1}{2}\right)}}\right)-1 / 2 \log \tilde{Y}\right\},
$$

where the tree level saddle point $h_{c}$ is given by the well known implicit equation given in (何). Inserting the results (13) and (15) in (3) with a redefinition of the constant

$$
\tilde{Y}_{c}=\tilde{Y}_{1}+\log \left(\frac{1}{h_{c}^{2}\left(1-h_{c}\right)^{2}} \sqrt{\frac{2 \pi \chi\left(\frac{1}{2}\right)}{\chi^{\prime \prime}\left(\frac{1}{2}\right)}}\right),
$$

we get the equation (6) announced in section I for the saturation critical line.

\section{DISCUSSION, OUTLOOK}

Let us describe general features of the critical line, when $\alpha$ is very small but otherwise not specified. Following expression (6), it is straightforward to realize that there is an absolute bound when $L, Y \gg 1$ given by $\tilde{Y} / 2 L>$ $\chi\left(\frac{1}{2}\right) / \chi^{\prime}\left(h^{*}\right) \sim .09$. Indeed below this bound, the compensation can no more take place between tree level and one loop contributions. It is interesting to note that the bound implies that a renormalization group evolution, with $L$ moving to large values while keeping $Y$ fixed, cannnot lead from a weak density to a saturation region, as expected from the general theoretical picture of the QCD dynamics of partons.

One can delimit three typical regimes in the saturation transition, depending upon the behaviour of the tree level amplitude:

I $0 \ll L_{0}->L_{1} \ll \tilde{Y}$ : in this region, saturation is obtained in the region $\tilde{Y}>\tilde{Y}_{0}+2 L\left(\frac{1}{2}-h^{*}\right) \sim \tilde{Y}_{0}+.63 L$, where $\tilde{Y}_{0}$ is a large effective rapidity scale incorporating the logarithmic prefactors.

II $L_{0} \ll L_{1}->L_{2}<\tilde{Y}$ : It is well-known that the BFKL behaviour discussed in I acquires an extra "diffusion term" (of the gluon transverse momenta along the BFKL ladder contributions) in the intermediate region. Within the saddle-point approximation it leads to the following saturation curve: 


$$
\tilde{Y}>\tilde{Y}_{0}+2 L\left(\frac{1}{2}-h^{*}\right)-\frac{\chi\left(\frac{1}{2}\right)}{\chi^{\prime \prime}\left(\frac{1}{2}\right)} \frac{2 L^{2}}{\tilde{Y}}
$$

where the last term is due to diffusion. Quite interestingly, saturation is favoured by diffusion. Indeed, formula (17) approximately interpolates between the behaviour I and II by a smooth decrease of the limiting slope of $\tilde{Y}$ versus $L$.

III $L_{2} \sim \tilde{Y}<L$ : near the bound $2 L / \tilde{Y}<\chi\left(\frac{1}{2}\right) / \chi^{\prime}\left(h^{*}\right) \sim 10$. In this region, one can use the relations

$$
h_{c} \sim \sqrt{\chi\left(\frac{1}{2}\right) \frac{2 L}{\tilde{Y}}} ; \quad \chi\left(h_{c}\right) \sim \frac{1}{h_{c}} .
$$

Using this and again introducing the effective large rapidity scale $\tilde{Y}_{0}$ one obtains, within a good approximation at large $(\tilde{Y}, L)$, the constraint

$$
\tilde{Y}>\left\{\sqrt{\frac{8 L}{\chi\left(\frac{1}{2}\right)}}+\sqrt{\frac{\tilde{Y}_{0}}{2}}\right\}^{2} .
$$

It is easy to check that (18) satisfies the bound $\tilde{Y} / 2 L>\chi\left(\frac{1}{2}\right) / \chi^{\prime}\left(h^{*}\right)$.

We have not yet discussed the rôle of the logarithmic prefactors. Using our explicit expressions, it will be easy to incorporate them in a more complete study which we leave for the future. They may play a phenomenological rôle. Indeed, it is important to note that the large factor $\frac{5}{2} \log \tilde{Y}$ of (6) is due to the double zeroes appearing in the loop couplings to the intermediate dipoles, see (A.25). This has the important effect of a strong energy dependent damping on the effective triple Pomeron couplings, which could otherwise be large [25]. Another possible important rôle would be, by mere extrapolation to higher dipole loops, to establish a useful hierarchy between these different orders. Indeed the coupling strength expected at order $n$, namely $\sim\left(\alpha^{2} \exp \omega\left(\frac{1}{2}\right) Y\right)^{n}$ cannot provide a hierarchical structure in the saturation regime. We are thus conjecturing a series of critical saturation lines corresponding to the onset of compensation between a growing number of higher loop order contributions. The study of this regime, necessary to understand the full saturation behaviour in the conformal invariant set-up will be pursued in the near future.

In fact, our work suggests a strategy to attack the full problem of saturation from dipole loops. It seems possible to analyze the $n$-loop dipole diagrams in the same way as we have shown here for the 2-loop case. It is clear that many diagrams can contribute, depending on the multiple rapidities where new dipoles can branch. However, we may conjecture that the integration over these intermediate rapidity intervals would dominantly lead to the QCD multi-Pomeron exchange and the corresponding $1 \rightarrow n$ dipole vertices which were analyzed in Ref. [28]. Then, it will be possible to explore the conformal invariant saturation regime it self and not only the transition to it.

Another point merits some care, when the onium typical sizes are of the same order. A quite interesting regime seems to set in when the sum (12) over all the double poles in the one-loop integrals becomes relevant. While being still at small coupling constant, this regime shares with the non-perturbative one the features of a summation over higher twist contributions. It would be nice to solve this case in detail.

Finally, the step between our calculations and actual experimental possibilities, which is not done in the present work, could be done either through factorization properties or by the consideration of "hard" subprocesses with very high energies like Mueller-Navelet jets at LHC or $\gamma^{*}-\gamma^{*}$ collisions at a future linear accelerator. Relevant studies are certainly deserved in the future.

\section{Acknowledgements}

We acknowledge fruitful discussions with Yuri Kovchegov, Heribert Weigert and Edmond Iancu. 


\section{APPENDIX 1}

\section{$S L(2, \mathcal{C})$-invariant formalism}

Using the $S L(2, \mathcal{C})$-invariant formalism, the solution of the BFKL equation obtained at leading log level of perturbative QCD [20] reads

$$
f_{Q}^{(0)}\left(\rho, \rho^{\prime} \mid Y\right)=\frac{\alpha^{2}}{4}\left|\rho \rho^{\prime}\right| \int d h \bar{E}_{Q}^{h}\left(\rho^{\prime}\right) E_{Q}^{h}(\rho) d(h) e^{\omega(h) Y},
$$

where the $S U(3)$ coupling $\alpha$ is fixed, small but unspecified, in the leading log approximation we consider.

In equation (A.1), the symbolic notation $\int d h \equiv \sum_{n=-\infty}^{\infty} \int d \nu$ corresponds to the integration over the quantum numbers associated to the continuous unitary irreducible representations of $S L(2, \mathcal{C})$, namely

$$
h=i \nu+\frac{1-n}{2} ; \tilde{h}=i \nu+\frac{1+n}{2} ; n \in \mathcal{N}, \nu \in \mathcal{R} .
$$

$E_{Q}^{h}(\rho)$ and $\omega(h)$ are, respectively, the $S L(2, \mathcal{C})$ Eigenfunctions and Eigenvalues of the BFKL kernel [23. The Eigenvalues read

$$
\omega(h)=\frac{\alpha N_{c}}{\pi} \chi(h) \equiv \frac{\alpha N_{c}}{\pi}\{2 \Psi(1)-\Psi(h)-\Psi(1-\tilde{h})\},
$$

where $\Psi \equiv(\log \Gamma)^{\prime}$. The $S L(2, \mathcal{C})$ Eigenvectors are defined

$$
E_{Q}^{h}(\rho)=\frac{2 \pi^{2}}{|\rho| b(h)} \int d^{2} r e^{i Q b} E^{h}\left(r-\frac{\rho}{2}, r+\frac{\rho}{2}\right),
$$

with

$$
E^{h}\left(r-\frac{\rho}{2}, r+\frac{\rho}{2}\right) \equiv \frac{|\rho| b(h)}{8 \pi^{4}} \int d^{2} r e^{-i Q r} E_{Q}^{h}(\rho)=(-)^{h-\tilde{h}}\left(\frac{\rho}{r^{2}-\frac{\rho^{2}}{4}}\right)^{h}\left(\frac{\bar{\rho}}{\bar{r}^{2}-\frac{\bar{\rho}^{2}}{4}}\right)^{\tilde{h}}
$$

where $\tilde{h}=1-\bar{h}, r$ is the 2-d impact-parameter, and introducing useful notations

$$
d(h)=\frac{1}{\left(\nu^{2}+\frac{(n-1)^{2}}{4}\right)\left(\nu^{2}+\frac{(n+1)^{2}}{4}\right)} ; b(h)=\frac{\pi^{3} 4^{h+\tilde{h}-1}}{\frac{1}{2}-h} \frac{\Gamma\left(-i \nu+\frac{1+|n|}{2}\right)}{\Gamma\left(i \nu+\frac{1+|n|}{2}\right)} \frac{\Gamma\left(i \nu+\frac{|n|}{2}\right)}{\Gamma\left(-i \nu+\frac{|n|}{2}\right)} ; a(h)=\frac{|b(h)|^{2}}{2 \pi^{2}}=\frac{\pi^{4} / 2}{\nu^{2}+\frac{n^{2}}{4}} .
$$

We will also make use of the formalism in the full coordinate space, the 4-diple amplitude $T^{(0)}$ being defined at tree level of QCD dipole contributions by

$$
T^{(0)}\left(\rho_{0}, \rho_{1} ; \rho_{0}^{\prime}, \rho_{1}^{\prime} \mid Y\right) \equiv \frac{1}{2 \pi} \int d^{2} Q e^{-i r Q} f_{Q}^{(0)}\left(\rho, \rho^{\prime} \mid Y\right),
$$

where the impact parameter is $r=\frac{1}{2}\left(\rho_{0}^{\prime}+\rho_{1}^{\prime}-\rho_{0}-\rho_{1}\right)$.

Inserting (A.1) into A.7), one finds

$$
T^{(0)}\left(\rho_{0}, \rho_{1} ; \rho_{0}^{\prime}, \rho_{1}^{\prime} \mid Y\right)=\frac{\alpha^{2}}{4} \int d h G^{h}\left(\rho_{0}, \rho_{1} ; \rho_{0}^{\prime}, \rho_{1}^{\prime}\right)\left(\nu^{2}+\frac{n^{2}}{4}\right) d(h) e^{\omega(h) Y},
$$

where, using the $S L(2, \mathcal{C})$ Eigenvectors in coordinate space (A.5), the Green function $G^{h}$ in coordinate space [21,23] can be written as

\footnotetext{
${ }^{1}$ Note that an analytic expression of the Eigenvectors $E_{Q}^{h}(\rho)$ in the mixed representation has been provided 21] in terms of a combination of products of two Bessel functions. For simplicity, we did not include the impact factors [23. 27]. Phenomenologically, the leading contribution to the amplitude (A.1) comes from the $n=0$ component which corresponds to the BFKL Pomeron.
} 


$$
G^{h}\left(\rho_{0}, \rho_{1} ; \rho_{0}^{\prime}, \rho_{1}^{\prime}\right) \equiv \int d^{2} \rho_{\alpha} \bar{E}^{h}\left(\rho_{0^{\prime} \alpha}, \rho_{1^{\prime} \alpha}\right) E^{h}\left(\rho_{0 \alpha}, \rho_{1 \alpha}\right)=\frac{|b(h)|^{2}}{\pi^{4}}\left|\rho \rho^{\prime}\right| \int d^{2} Q e^{-i b Q} \bar{E}_{Q}^{h}\left(\rho^{\prime}\right) E_{Q}^{h}(\rho) .
$$

We will also define for convenience the "instantaneous" (i.e. Y=0) amplitude

$$
T\left(\rho_{0}, \rho_{1} ; \rho_{0}^{\prime}, \rho_{1}^{\prime}\right) \equiv T^{(0)}\left(\rho_{0}, \rho_{1} ; \rho_{0}^{\prime}, \rho_{1}^{\prime} \mid Y=0\right) .
$$

Due to completeness and frame independence of the formalism, many formulae can be simplified using these "instantaneous" amplitudes between dipoles.

The single multiplicity distribution of dipoles $n_{1}$ after an evolution characterized by the rapidity $Y$ is simply related [21] to the previous expressions (e.g. (A.9)), namely

$$
n_{1}\left(\rho_{0}, \rho_{1} ; \rho_{0}^{\prime}, \rho_{1}^{\prime} \mid Y\right)=\frac{1}{\pi^{2}\left|\rho^{\prime}\right|^{2}} \int d h \quad G^{h}\left(\rho_{0}, \rho_{1} ; \rho_{0}^{\prime}, \rho_{1}^{\prime}\right)\left(\nu^{2}+n^{2} / 4\right) e^{\omega(h) Y} .
$$

Let us finally note a useful completeness formula [21] valid at tree level:

$$
\begin{aligned}
T^{(0)}\left(\rho_{0} \rho_{1} ; \rho_{0}^{\prime} \rho_{1}^{\prime} \mid Y=y+y_{a}+y^{\prime}\right) & \equiv \frac{1}{2^{2}(2 \pi)^{4}} \int \frac{d^{2} \rho_{a_{0}} d^{2} \rho_{a_{1}}}{\left|\rho_{a}\right|^{2}} \frac{d^{2} \rho_{a_{0}^{\prime}} d^{2} \rho_{a_{1}^{\prime}}}{\left|\rho_{a^{\prime}}\right|^{2}} \\
& \times n_{1}\left(\rho_{0} \rho_{1} ; \rho_{a_{0}} \rho_{a_{1}} \mid y\right) \bar{n}_{1}\left(\rho_{0}^{\prime} \rho_{1}^{\prime} ; \rho_{a_{0}}^{\prime} \rho_{a_{1}}^{\prime} \mid y^{\prime}\right) T^{(0)}\left(\rho_{a_{0}} \rho_{a_{1}}, \rho_{a_{0}^{\prime}} \rho_{a_{1}^{\prime}} \mid y_{a}\right),
\end{aligned}
$$

where $\rho_{0}, \rho_{1}$ are the transverse coordinates of one initial dipole (resp. $\rho_{0}^{\prime} \rho_{1}^{\prime}$ for the second one), $\rho_{a_{0}}, \rho_{a_{1}}$ are those of the interacting dipole emerging from the dipole $\rho_{0}, \rho_{1}$ after evolution in rapidity $y$ (resp. $\rho_{a_{0}^{\prime}}, \rho_{a_{1}^{\prime}}$ and $y^{\prime}$ for the second one), which interaction is described by the amplitude $T\left(\rho_{a_{0}}, \rho_{a_{1}} ; \rho_{a_{0}^{\prime}}, \rho_{a_{1}^{\prime}} \mid y_{a}\right)$ as in (A.8).

Equation (A.12) has the physical interpretation that, at tree dipole level, the multiplication of dipoles due to rapidity evolution does not lead to saturation, but to the same tree level amplitude in a kind of bootstrap property. 


\section{APPENDIX 2}

\section{The two-dipole density distribution $n_{2}$}

The two-dipole density distribution $n_{2}$ results from the solution of a specific evolution equation which consists of an extension of the mixed evolution of the one formulated in ref. [4, 31,24, namely:

$$
\begin{aligned}
n_{2} & \left(\rho_{0} \rho_{1} ; \rho_{a_{0}} \rho_{a_{1}}, \rho_{b_{0}} \rho_{b_{1}} \mid y-\bar{y}, \bar{y}\right)= \\
& =\frac{\alpha N_{c}}{2 \pi^{2}} \int \frac{d h d h_{a} d h_{b}}{\left|\rho_{a} \rho_{b}\right|^{2}} \int d \omega_{1} \frac{\mathbf{e}^{\omega_{1}(y-\bar{y})}}{\omega_{1}-\omega(h)} \int d \omega \frac{e^{\omega y}}{\omega\left(h_{a}\right)+\omega\left(h_{b}\right)-\omega} n_{2}^{h, h_{a}, h_{b}}\left(\rho_{0} \rho_{1} ; \rho_{a_{0}} \rho_{a_{1}}, \rho_{b_{0}} \rho_{b_{1}}\right),
\end{aligned}
$$

where, by convention, $d \omega_{i} \rightarrow d \omega_{i} / 2 \pi$ and

$$
\begin{aligned}
n_{2}^{h, h_{a}, h_{b}}\left(\rho_{0} \rho_{1} ; \rho_{a_{0}} \rho_{a_{1}}, \rho_{b_{0}} \rho_{b_{1}}\right)=\frac{1}{a(h) a\left(h_{a}\right) a\left(h_{b}\right)} & \quad \int d^{2} \rho_{\alpha} d^{2} \rho_{\beta} d^{2} \rho_{\gamma} \overline{\mathcal{R}}_{\alpha, \beta, \gamma}^{h, h_{a}, h_{b}} \times \\
& \times E^{h_{a}}\left(\rho_{a_{0} \alpha}, \rho_{a_{1} \alpha}\right) E^{h_{b}}\left(\rho_{b_{0} \beta}, \rho_{b_{1} \beta}\right) E^{h}\left(\rho_{0 \gamma}, \rho_{1 \gamma}\right),
\end{aligned}
$$

with

$$
\mathcal{R}_{\alpha, \beta, \gamma}^{h, h_{a}, h_{b}} \equiv \int \frac{d^{2} r_{0} d^{2} r_{1} d^{2} r_{2}}{\left|r_{01} r_{02} r_{12}\right|^{2}} E^{h}\left(r_{0 \gamma}, r_{1 \gamma}\right) E^{h_{a}}\left(r_{0 \alpha}, r_{2 \alpha}\right) E^{h_{b}}\left(r_{1 \beta}, r_{2 \beta}\right)
$$

where $\rho=\rho_{0}-\rho_{1}, \rho_{a}=\rho_{a_{0}}-\rho_{a_{1}}, \rho_{b}=\rho_{b_{0}}-\rho_{b_{1}}$.

Conformal invariance implies the well-known tensorial reduction [29]

$$
\mathcal{R}_{\alpha, \beta, \gamma}^{h, h_{a}, h_{b}} \equiv\left[\rho_{\alpha \beta}\right]^{h-h_{a}-h_{b}}\left[\rho_{\beta \gamma}\right]^{h_{a}-h_{b}-h}\left[\rho_{\gamma \alpha}\right]^{h_{b}-h_{a}-h}\left[\bar{\rho}_{\alpha \beta}\right]^{\tilde{h}_{-} \tilde{h}_{a}-\tilde{h}_{b}}\left[\bar{\rho}_{\beta \gamma}\right]^{\tilde{h}_{a}-\tilde{h}_{b}-\tilde{h}}\left[\bar{\rho}_{\gamma \alpha}\right]^{\tilde{h}_{b}-\tilde{h}_{a}-\tilde{h}} g_{3 \mathcal{P}}\left(h, h_{a}, h_{b}\right),
$$

where $g_{3 \mathcal{P}}\left(h, h_{a}, h_{b}\right)$ happens to be [25] the QCD triple Pomeron coupling as obtained in the dipole formulation, namely:

$$
g_{3 \mathcal{P}}\left(h, h_{a}, h_{b}\right)=\int \frac{d^{2} r_{0} d^{2} r_{1} d^{2} r_{2}}{\left|r_{01} r_{02} r_{12}\right|^{2}}\left[r_{01}\right]^{h}\left[\frac{r_{02}}{r_{0} r_{2}}\right]^{h_{a}}\left[\frac{r_{12}}{\left(1-r_{1}\right)\left(1-r_{2}\right)}\right]^{h_{b}}\left[\bar{r}_{01}\right]^{\tilde{h}}\left[\frac{\bar{r}_{02}}{\bar{r}_{0} \bar{r}_{2}}\right]^{\tilde{h}_{a}}\left[\frac{\bar{r}_{12}}{\left(1-\bar{r}_{1}\right)\left(1-\bar{r}_{2}\right)}\right]^{\tilde{h}_{b}} .
$$

Considering the Fourier transforms (A.4) for the $S L(2, \mathcal{C})$ Eigenvectors $E_{q}^{h}$ in the mixed representation, one writes

$$
\begin{aligned}
n_{2}^{h, h_{a}, h_{b}}\left(\rho_{0} \rho_{1} ; \rho_{a_{0}} \rho_{a_{1}}, \rho_{b_{0}} \rho_{b_{1}}\right) & =g_{3 \mathcal{P}}\left(h, h_{a}, h_{b}\right) \frac{b(h) b\left(h_{a}\right) b\left(h_{b}\right)}{2^{7} \pi^{10}}\left|\rho \rho_{a} \rho_{b}\right| \int d^{2} q_{a} d^{2} q_{b} d^{2} Q \delta^{(2)}\left(Q+q_{a}+q_{b}\right) \\
& \times E_{Q}^{h}(\rho) E_{q_{a}}^{h_{a}}\left(\rho_{a}\right) E_{q_{b}}^{h_{b}}\left(\rho_{b}\right) e^{-i\left(q_{a} r_{a}+q_{b} r_{b}+Q r\right)} \int d^{2} v d^{2} w e^{-i\left(\left(q_{a}-q_{b}\right) \frac{v}{2}+Q \frac{w}{2}\right)} \\
& \times\left\{[v]^{-1-h-h_{a}-h_{b}}[(w-v) / 2]^{-1+h-h_{a}+h_{b}}[(w+v) / 2]^{-1+h+h_{a}-h_{b}}\right\} \times\{a \cdot h \cdot\}
\end{aligned}
$$

where $\rho_{\alpha \beta}=v, \rho_{\alpha \gamma}+\rho_{\beta \gamma}=w, 2 r_{a}=\rho_{a 0}+\rho_{a 1}, 2 r_{b}=\rho_{b 0}+\rho_{1}$ and $r$ is the overall impact parameter. The notation $\{a . h$.$\} indicates the anti-holomorphic part of the bracketed term in the integrand for which the integration variables$ are complex conjugates and the exponents $h_{i}$ are replaced by $\tilde{h}_{i}$. Equivalently, the distribution $n_{2}$ for the lower vertex is given by the same equation (A.18) by using prime indices. 


\section{APPENDIX 3}

\section{The general one-loop amplitude}

Implementing equations A.8 and A.18 in the master equation (3) and first integrating over impact parameters, one gets:

$$
(2 \pi)^{8} \delta\left(q_{a}-q^{\prime \prime}\right) \delta\left(q_{b}-q^{\prime \prime}{ }_{b}\right) \delta\left(q_{a}^{\prime}-q^{\prime \prime}{ }_{a}\right) \delta\left(q_{b}^{\prime}-q^{\prime \prime}{ }_{b}\right)
$$

Then, integrating over the intermediate dipole sizes, and using the orthogonality [23] relation

$$
\frac{1}{4 \pi^{2}} \int \frac{d^{2} \rho}{|\rho|^{2}} E_{q}^{h}(\rho) \bar{E}_{q}^{h^{\prime}}(\rho)=\delta^{(2)}\left(h, h^{\prime}\right)+\delta^{(2)}\left(h, 1-h^{\prime}\right) e^{i \phi(h)}
$$

where $e^{i \phi(h)} \equiv E_{q}^{h}(\rho) / E_{q}^{1-h}(\rho)$ is just a $\rho, q$-independent phase factor given in Ref. 23, one finds

$$
(2 \pi)^{8} 2^{4} \delta^{(2)}\left(h_{a}, h^{\prime \prime}\right) \delta^{(2)}\left(h_{b}, h^{\prime \prime}\right) \delta^{(2)}\left(h_{a}^{\prime}, 1-h^{\prime}{ }_{a}\right) \delta^{(2)}\left(h_{b}^{\prime}, 1-h^{\prime \prime}\right),
$$

where $\delta^{(2)}\left(h, h^{\prime}\right) \equiv \delta_{n n^{\prime}} \delta\left(\nu-\nu^{\prime}\right)$. The integration over $q_{a}-q_{b}$ finally gives $(2 \pi)^{2} \delta\left(v-v^{\prime}\right)$.

Plugging in formulae (8,9) the result obtained in formula (A.18) and integrating over $\delta$-fuctions, one gets:

$$
\begin{array}{r}
f_{Q}^{(1)}\left(\rho, \rho^{\prime} \mid Y=y+y^{\prime}\right)=-\frac{\alpha^{4} \pi^{2}}{8}\left(\frac{\alpha N_{c}}{2 \pi^{2}}\right)^{2} \int d h_{a} d h_{b} \frac{d\left(h_{a}\right)}{\left|b\left(h_{a}\right)\right|^{2}} \frac{d\left(h_{b}\right)}{\left|b\left(h_{b}\right)\right|^{2}} \\
\times \int d h d h^{\prime} \frac{g_{3 \mathcal{P}}\left(h, h_{a}, h_{b}\right)}{b(1-h)} \frac{g_{3 \mathcal{P}}^{*}\left(h^{\prime}, h_{a}, h_{b}\right)}{b\left(h^{\prime}\right)}\left|\rho \rho^{\prime}\right| E_{Q}^{h}(\rho) \bar{E}_{Q}^{h^{\prime}}\left(\rho^{\prime}\right) \mathcal{H}_{Q}\left(h, h^{\prime}\right) \\
\times \int_{0}^{y} d \bar{y} \int_{0}^{Y-y^{\prime}} d \bar{y}^{\prime} \int d \omega d \omega_{1} d \omega^{\prime} d \omega_{1}^{\prime} \frac{e^{\omega \bar{y}+\omega_{1}(y-\bar{y})}}{\left(\omega\left(h_{a}\right)+\omega\left(h_{b}\right)-\omega\right)\left(\omega_{1}-\omega(h)\right)} \frac{e^{\omega^{\prime} \bar{y}^{\prime}+\omega_{1}^{\prime}\left(y^{\prime}-\bar{y}^{\prime}\right)}}{\left(\omega\left(h_{a}\right)+\omega\left(h_{b}\right)-\omega^{\prime}\right)\left(\omega_{1}^{\prime}-\omega\left(h^{\prime}\right)\right)}
\end{array}
$$

where, after the change of variables $w=v(1-2 t), w^{\prime}=v\left(1-2 t^{\prime}\right)$,

$$
\mathcal{H}_{Q}\left(h, h^{\prime}\right)=\int d^{2} v d^{2} t d^{2} t^{\prime} e^{i Q v\left(t-t^{\prime}\right)}\left\{v^{h-h^{\prime}-1} t^{-1+h+h_{b}-h_{a}}(1-t)^{-1+h-h_{b}+h_{a}} t^{\prime h_{a}-h_{b}-h^{\prime}}\left(1-t^{\prime}\right)^{-h_{a}+h_{b}-h^{\prime}}\right\} \times a . h .
$$

After integration over $v$ one obtains 30

$$
\begin{aligned}
\mathcal{H}_{Q}\left(h, h^{\prime}\right) & =\left(\frac{2}{\bar{Q}}\right)^{h-h^{\prime}}\left(\frac{2}{Q}\right)^{\tilde{h}-\tilde{h}^{\prime}} e^{i \frac{\pi}{2}\left(n-n^{\prime}\right)} \gamma\left(h-h^{\prime}\right) \int d^{2} t d^{2} t^{\prime}\left(t-t^{\prime}\right)^{h^{\prime}-h} \\
& \times\left\{t^{-1+h+h_{b}-h_{a}}(1-t)^{-1+h-h_{b}+h_{a}} t^{\prime h_{a}-h_{b}-h^{\prime}}\left(1-t^{\prime}\right)^{-h_{a}+h_{b}-h^{\prime}}\right\} \times\{a . h .\} .
\end{aligned}
$$

where, by definition, $\gamma(z) \equiv \Gamma(z) / \Gamma(1-\tilde{z})$.

The remaining integral can be proven ${ }^{2}$ to reduce to

$\mathcal{H}_{Q}\left(h, h^{\prime}\right)=\left(\frac{2}{\bar{Q}}\right)^{h-h^{\prime}}\left(\frac{2}{Q}\right)^{\tilde{h}-\tilde{h}^{\prime}} \frac{\pi^{2}}{(2 h-1)(2 \tilde{h}-1)}\left[\delta\left(h-h^{\prime}\right)+(-)^{n+n_{a}+n_{b}} \gamma\left(h+h_{a}-h_{b}\right) \gamma\left(1-h^{\prime}-h_{a}+h_{b}\right) \delta\left(1-h-h^{\prime}\right)\right]$

The integral over $h^{\prime}$ can now easily be performed. The remaining poles at $h^{\prime}=h$ and $h^{\prime}=1-h$ give twice the same contribution due to the over completeness relation of the $E_{Q}^{h}$ generators [23]. One finally obtains:

\footnotetext{
${ }^{2}$ One shows using general methods [30] that $\mathcal{H}_{Q}\left(h, h^{\prime}\right)$ is a combination of $\delta$ functions whose coefficients can then be computed directly on formula (A.22).
} 


$$
\begin{gathered}
f_{Q}^{(1)}\left(\rho, \rho^{\prime} \mid Y\right)=-\frac{\alpha^{4} \pi^{4}}{8}\left(\frac{\alpha N_{c}}{2 \pi^{2}}\right)^{2} \int d h d h_{a} d h_{b}\left|\frac{g_{3 \mathcal{P}}\left(h, h_{a}, h_{b}\right)}{b\left(h_{a}\right) b\left(h_{b}\right) b(h)}\right|^{2} d\left(h_{a}\right) d\left(h_{b}\right) \frac{(-1)^{n+n_{a}+n_{b}}}{\left|\frac{1}{2}-h\right|^{2}}\left|\rho \rho^{\prime}\right| E_{Q}^{h}(\rho) \bar{E}_{Q}^{h}\left(\rho^{\prime}\right) \\
\times \int_{0}^{y} d \bar{y} \int_{0}^{Y-y} d \bar{y}^{\prime} \int d \omega d \omega_{1} d \omega^{\prime} d \omega_{1}^{\prime} \frac{e^{\omega \bar{y}+\omega_{1}(y-\bar{y})}}{\left(\omega\left(h_{a}\right)+\omega\left(h_{b}\right)-\omega\right)\left(\omega_{1}-\omega(h)\right)} \frac{e^{\omega^{\prime} \bar{y}^{\prime}+\omega_{1}^{\prime}\left(y^{\prime}-\bar{y}^{\prime}\right)}}{\left(\omega\left(h_{a}\right)+\omega\left(h_{b}\right)-\omega^{\prime}\right)\left(\omega_{1}^{\prime}-\omega(h)\right)} .
\end{gathered}
$$

This one-loop amplitude preserves the global conformal invariance of the tree-level BFKL 4-gluon amplitude, since the only scale-dependence on $Q$ is present in the conformal Eigenvectors $\left|\rho \rho^{\prime}\right| E_{Q}^{h}(\rho) \bar{E}_{Q}^{h}\left(\rho^{\prime}\right)$.

It was noted in Ref. 22] that the integration over rapidity variables yields two different contributions depending on the sign of the quantity $\omega\left(h_{a}\right)+\omega\left(h_{b}\right)-\omega(h)$. Indeed for $\omega\left(h_{a}\right)+\omega\left(h_{b}\right)<\omega(h)$, the relevant poles are situated at $\omega=\omega_{1}=\omega^{\prime}=\omega_{1}^{\prime}=\omega(h)$, leading to a contribution associated with the single Pomeron dependence $e^{\omega(h) Y}$. In the opposite case, namely $\omega\left(h_{a}\right)+\omega\left(h_{b}\right)>\omega(h)$, the relevant poles are situated at $\omega=\omega_{1}=\omega^{\prime}=\omega_{1}^{\prime}=\omega\left(h_{a}\right)+\omega\left(h_{b}\right)$. The resulting amplitude

$$
\begin{aligned}
f_{Q}^{(1)}\left(\rho, \rho^{\prime} \mid Y\right) \sim & -\frac{1}{2 \pi^{8}}\left(\frac{\alpha}{2 \pi^{2}}\right)^{4}\left|\rho \rho^{\prime}\right| \int d h(-1)^{n} \bar{E}_{Q}^{h}\left(\rho^{\prime}\right) E_{Q}^{h}(\rho) \\
& \times \iint d h_{a} d h_{b}\left[\frac{\left(\frac{1}{2}-h_{a}\right)\left(\frac{1}{2}-h_{b}\right)}{h_{a}\left(1-h_{a}\right) h_{b}\left(1-h_{b}\right)}\right]^{2} e^{\left(\omega\left(h_{a}\right)+\omega\left(h_{b}\right)\right) Y}\left|\frac{g_{3 \mathcal{P}}\left(h, h_{a}, h_{b}\right)}{\chi(h)-\chi\left(h_{a}\right)-\chi\left(h_{b}\right)}\right|^{2},
\end{aligned}
$$

is the one we are interested in for the saturation problem since it possesses the QCD "double Pomeron" energy behaviour $e^{\left(\omega\left(h_{a}\right)+\omega\left(h_{b}\right)\right) Y}>e^{\omega(h) Y}$. This is precisely the energy behaviour which will compensate for the higher order $\alpha^{2}$ in the coupling constant. Notice that the expression depends only on the sum $Y=y+y^{\prime}$, as it should do from longitudinal boost invariance. 
[1] L.V. Gribov, E.M. Levin and M.G.Ryskin, Phys. Rep. 100 (1983) 1.

[2] A.H.Mueller, J.Qiu, Nucl. Phys. B268 (1986) 427.

[3] N.N.Nikolaev, B.G.Zakharov, Z.Phys. C49 (1991) 607, Z.Phys C53 (1992) 331, Z.Phys. C64 (1994) 651, J.Exp.Theor.Phys. 78 (1994) 598.

[4] A.H. Mueller, Nucl.Phys. B415 (1994) 373; A.H. Mueller and B. Patel, Nucl.Phys. B425 (1994) 471; A.H. Mueller, Nucl.Phys. B437 (1995) 107.

[5] A.H. Mueller, Nucl.Phys. B335 (1990) 115; Yu.A. Kovchegov, A.H. Mueller and S. Wallon, Nucl.Phys. B507 (1997) 367; A.H. Mueller, Eur.Phys.J. A1 (1998) 19, Nucl.Phys. A654 (1999) 370, Nucl.Phys. B558 (1999) 285.

[6] J.C.Collins and J.Kwiecinski, Nucl.Phys. B335 (1990) 89; J. Bartels, G.A. Schuler and J. Blümlein, Z.Phys. C50 (1991) 91, Nucl.Phys.Proc.Suppl. 18 (1991) 147.

[7] J. Bartels and E.M. Levin, Nucl.Phys. B387 (1992) 617; J. Bartels, Phys.Lett. B298 (1993) 204, Z.Phys. C60 (1993) 471, Z.Phys. C62 (1994) 425;

J. Bartels and M .Wüsthoff, Z.Phys. C66 (1995) 157;

J. Bartels and C.Ewertz, JHEP 9909 (1999) 026.

[8] L. McLerran and R. Venugopalan, Phys.Rev. D49 (1994) 2233, Phys.Rev. D49 (1994) 3352, Phys.Rev. D50 (1994) 2225 ;

A. Kovner, L. McLerran and H. Weigert, Phys.Rev. D52 (1995) 6231, Phys.Rev. D52 (1995) 3809;

R. Venugopalan, Acta Phys.Polon. B30 (1999) 3731;

E. Iancu and L. McLerran, Phys.Lett. B510 (2001) 145;

L. McLerran, The Color Glass Condensate and Small x Physics: 4 Lectures hep-ph/0104285;

E. Iancu, A. Leonidov and L. McLerran, Nucl.Phys. A692 (2001) 583;

E. Ferreiro, E. Iancu, A. Leonidov and L. McLerran, Nonlinear Gluon Evolution in the Color Glass Condensate: II hep-ph/0109115;

A. Capella, et al. Phys.Rev. D63 (2001) 054010.

[9] G.P. Salam, Nucl.Phys. B449 (1995) 589, Nucl.Phys. B461 (1996) 512, Comput.Phys.Commun. 105 (1997) 62;

G.P. Salam and A.H.Mueller, Nucl.Phys. B475 (1996) 293.

[10] E. Gotsman, E.M. Levin and U. Maor, Nucl.Phys. B464 (1996) 251, Nucl.Phys. B493 (1997) 354, Phys.Lett. B245 (1996) 369, Eur.Phys.J. C5 (1998) 303; E. Gotsman, E.M. Levin, U. Maor and E. Naftali, Nucl.Phys. B539 (1999) 535; A.L. Ayala Filho, M.B. Gay Ducati and E.M. Levin, Nucl.Phys. B493 (1997) 305, Nucl.Phys. B551 (1998) 355, Eur.Phys.J. C8 (1999) 115.

[11] I. Balitsky, Nucl.Phys. B463 (1996) 99; High-Energy QCD and Wilson Lines To be published in the Boris Ioffe Festschrift "At the Frontier of Particle Physics/Handbook of QCD", edited by M. Shifman (World Scientific, Singapore, 2001)hep-ph/0101042.

[12] J. Jalilian-Marian, A. Kovner, L. McLerran and H. Weigert, Phys.Rev. D55 (1997) 5414; J. Jalilian-Marian, A. Kovner and H. Weigert, Phys.Rev. D59 (1999) 014014, Phys.Rev. D59 (1999) 014015, Phys.Rev. D59 (1999) 034007, Erratum - ibid. D59 (1999) 099903; A. Kovner, J. Guilherme Milhano and H. Weigert, Phys.Rev. D62 (2000) 114005.

[13] H. Weigert, Unitarity at small Bjorken $x$ hep-ph/0004044.

[14] M.A. Braun, Eur.Phys.J. C16 (2000) 337hep-ph/0101070.

[15] Yu.A. Kovchegov and L. McLerran, Phys.Rev. D60 (1999) 054025; Erratum - ibid. Phys.Rev. D62 (2000) 019901; Yu.A. Kovchegov and E.M. Levin, Phys.Rev. D60 (1999) 034008; Yu.A. Kovchegov, Phys.Rev. D59 (1999) 014017, Phys.Rev. D61 (2000) 074018.

[16] E.M. Levin and K. Tuchin, Nucl.Phys. B537 (2000) 99, Nucl.Phys. A691 (2001) 779, Nucl.Phys. A693 (2001) 787.

[17] K. Golec-Biernat and M. Wüsthoff, Phys.Rev. D59 (1998) 014017

[18] N. Timneanu, J. Kwiecinski, L. Motyka, Saturation model for two-photon interactions at high energies hep-ph/0110409.

[19] S. Munier, A.M. Stasto, A.H. Mueller, Nucl.Phys. B603 (2001) 427.

[20] L.N.Lipatov, Sov. J. Nucl. Phys. 23 (1976) 642; V.S.Fadin, E.A.Kuraev and L.N.Lipatov, Phys. lett. B60 (1975) 50; E.A.Kuraev, L.N.Lipatov and V.S.Fadin, Sov.Phys.JETP 44 (1976) 45, 45 (1977) 199; I.I.Balitsky and L.N.Lipatov, Sov.J.Nucl.Phys. 28 (1978) 822.

[21] H.Navelet, R.Peschanski, Nucl. Phys. B507 (1997) 353; H.Navelet, S.Wallon, Nucl. Phys. B522 (1998) 237.

[22] H.Navelet and R.Peschanski, Phys. Rev. Lett. 82 (1999) 1370.

[23] L.N.Lipatov, Zh. Eksp. Teor. Fiz. 90 (1986) 1536 (Eng. trans. Sov. Phys. JETP 63 (1986) 904); Phys. Rept. 286 (1997) 131.

[24] R.Peschanski, Phys. lett. B409 (1997) 491.

[25] A.Bialas, H.Navelet, R.Peschanski, Phys. Rev. D57 (1998) 6585;

G.P.Korchemsky, Nucl. Phys. B550 (1999) 397. 
[26] V.A Abramovsky, V.N.Gribov, O.V.Kancheli, Sov. J. Nucl. Phys. 18 (1974) 308; J.Bartels, M.G.Ryskin Zeit. Phys. C76 1997241.

[27] H.Navelet, R.Peschanski, Nucl. Phys. B515 (1998) 269.

[28] R.A.Janik, R. Peschanski, Nucl.Phys. B549 (1999) 280.

[29] A.Polyakov, Pisma Zh. Eksp. Teor. Fiz. 12 (1970) 538.

[30] J.S.Geronimo, H.Navelet, math-ph/0003019, to appear in Journ. of Math. Phys.

[31] A.Bialas, R.Peschanski, Phys. lett. B355 (1995) 301. 


\section{FIGURES}

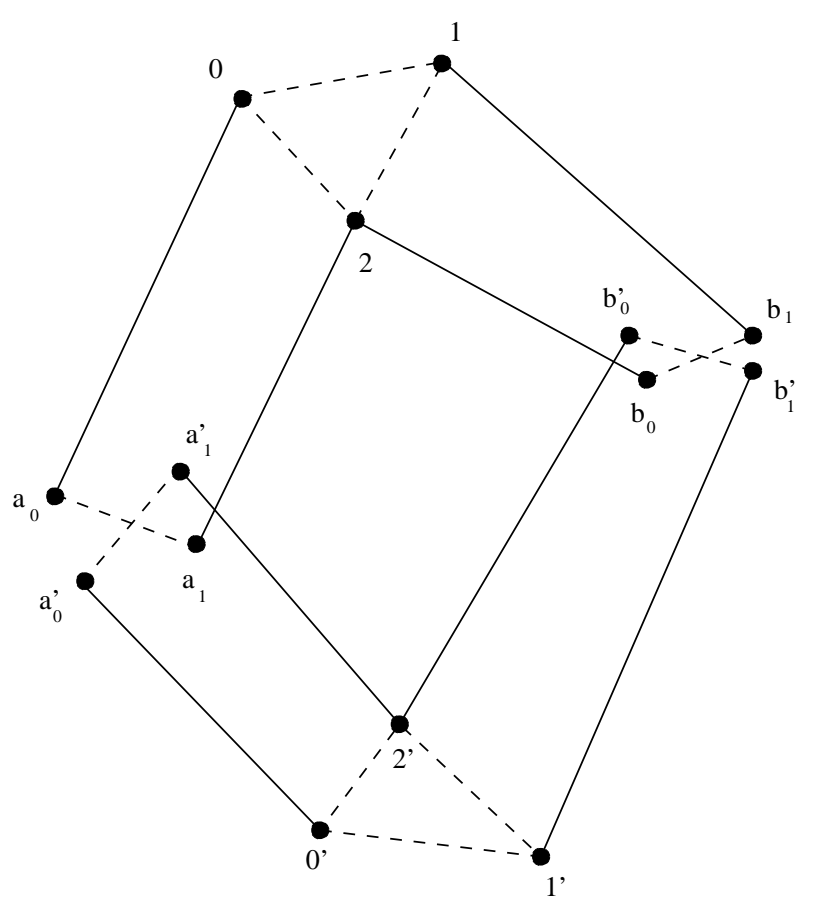

Figure 1

One-loop dipole amplitude in transverse coordinate space

The transverse coordinates $\rho_{i}$ of the incident and of the two interacting dipoles are simply denoted by their respective indices $i$. The coordinates $2,2^{\prime}$ refer to the integration points, see Appendix 2.

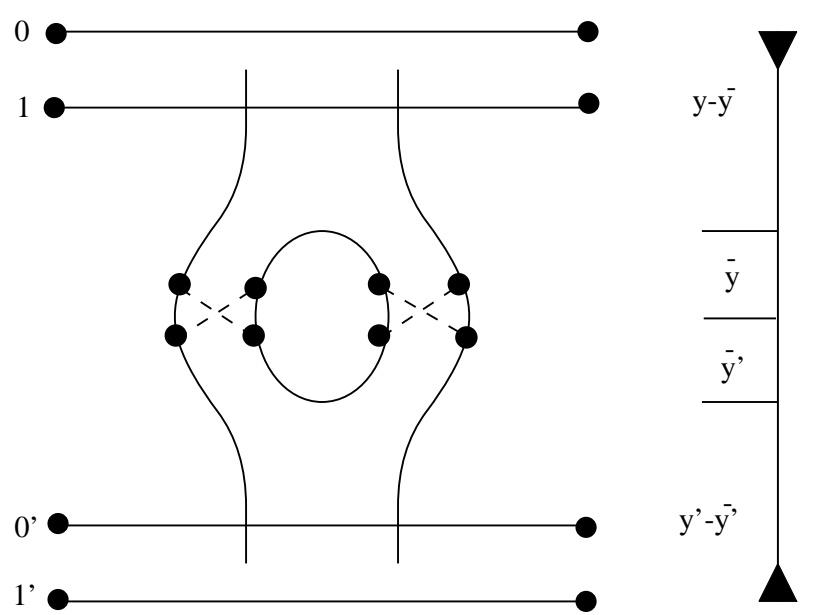

Figure 2

One-loop dipole amplitude in rapidity space

The initial dipoles $\rho_{0} \rho_{1}, \rho_{0}^{\prime} \rho_{1}^{\prime}$ evolve in the rapidity range $y-$ $\bar{y}^{\prime}, y^{\prime}-\bar{y}^{\prime}$ with one-Pomeron type of evolution and then with twoPomeron type of evolution over the range $\bar{y}, \bar{y}^{\prime}$. Note that $y+y^{\prime} \equiv$ $Y$, is the total rapidity range. After integration over $\bar{y}, \bar{y}^{\prime}$, see Appendix 3, the resulting amplitude depends on the total range $Y$. 


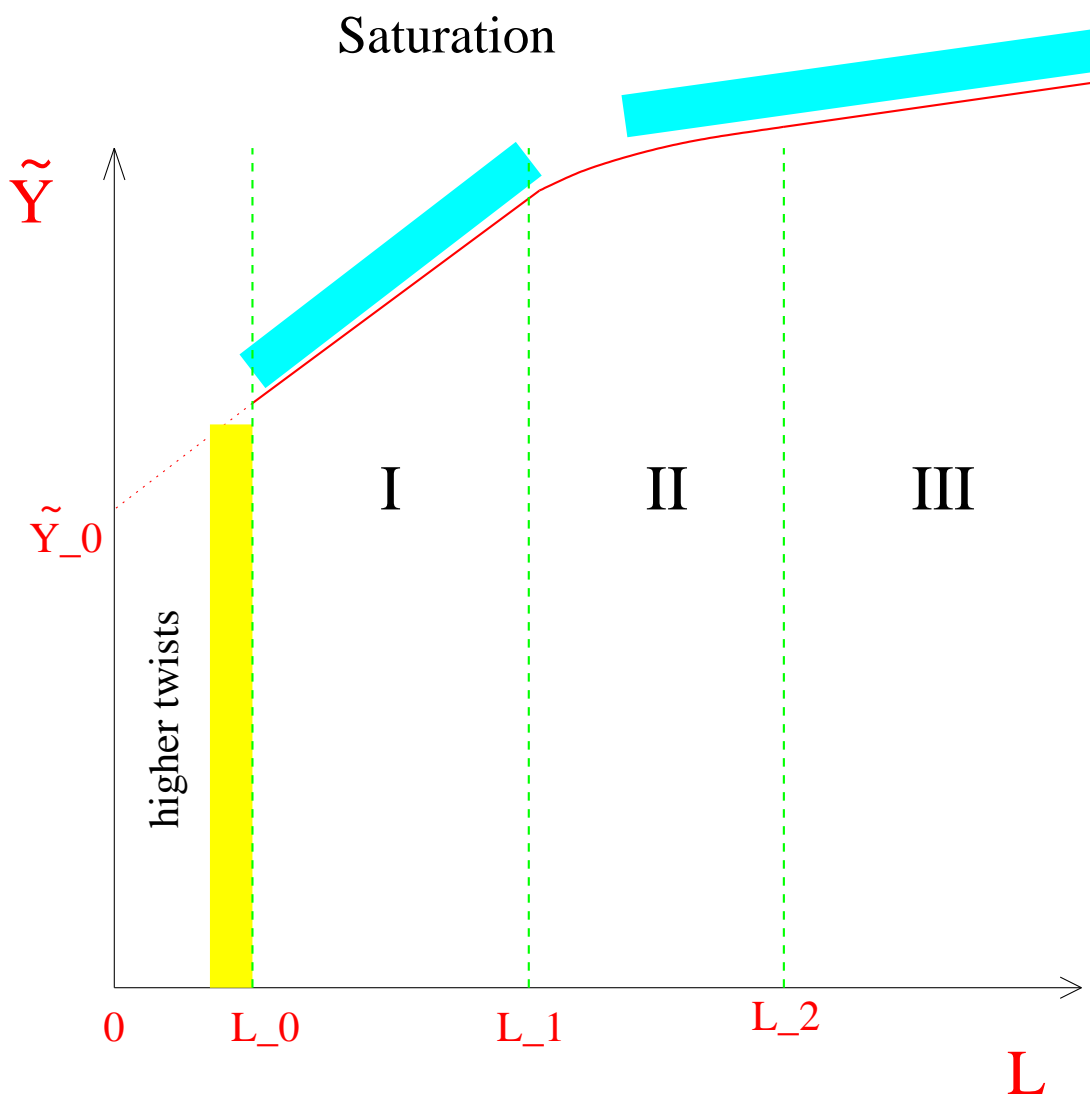

Figure 3

Critical Saturation line

The critical saturation line is represented in the plane $\tilde{Y}, L$. The saturation region is under the curve. The region beyond the validity of leading twist approximation is below a value $1<<L_{0}$. The regions I, II, III correspond to different regimes of compensation between tree and one-loop dipole contributions (see text). 